\title{
PROCESSO DO TRABALHO EM SAÚDE NA ATENÇÃO PRIMÁRIA: UM PASSEIO PELA LITERATURA
}

\author{
Aydwlha Moniq Barbosa SANTANA ${ }^{1}$ \\ Felipe Gomes de ASSIS ${ }^{2}$ \\ Luciana Dantas Farias de ANDRADE ${ }^{3}$ \\ Priscyla Rocha de Brito LIRA ${ }^{4}$
}

\begin{abstract}
${ }^{1}$ Enfermeira. Especialista em Saúde da Família, aydwlham@gmail.com
${ }^{2}$ Enfermeiro. Especialista em Saúde da Família, euartur01@gmail.com

${ }^{3}$ Orientadora. Professora do Curso de Graduação em Enfermagem da UFCG - Campus Cuité. Paraíba. Doutora em

Psicologia. luciana_dantas_farias@yahoo.com.br

${ }^{4}$ Enfermeira, priscyla.lira@hotmail.com
\end{abstract}

\section{Recebido em: 13/08/2013 - Aprovado em: 21/12/2013 - Disponibilizado em: 15/01/2014}

RESUMO: O processo de trabalho é compreendido como o fazer, isto é, o pôr em prática. Sendo o processo de trabalho em saúde um enumerado de atividades, que o profissional sozinho ou juntamente com a equipe realizam atividades de cunho social, esse trabalho pode ser físico, intelectual, ou ainda a junção das duas para mudar ou transformar algo ou alguma coisa. Desta forma, este estudo teve como objetivo Realizar uma revisão da literatura sobre processo do trabalho em saúde na atenção primária. O presente estudo trata-se de revisão da literatura que seguiu as seguintes etapas: escolha do tema, pesquisa de artigos publicados em periódicos científicos nacionais e bibliotecas eletrônicas disponíveis na Scientific Electronic Library Online (Scielo), e (Dedalus), elaboração do plano de trabalho, elaboração do texto, para tanto levou-se em consideração o Código de Ética dos Profissionais de Enfermagem. O trabalho em saúde está concomitantemente relacionado ao trabalho em equipe, pois o mesmo exige das pessoas um trabalho coletivo e para desenvolverem juntos um trabalho profissional, todos da equipe necessitam relacionar-se e para, a partir daí desenvolverem projetos para um bom atendimento. Portanto, a organização do processo de trabalho em saúde na atenção primária deve promover suficientemente o vínculo e responsabilização com o usuário dos serviços, favorecendo a busca de atendimento quando necessário.

Palavra chaves: Atenção Primária à Saúde. Avaliação de Processos. Saúde da Família. Modelo de Atenção. Saúde.

\section{PROCESS OF WORKING IN HEALTH IN PRIMARY CARE: A RIDE FOR LITERATURE}

\begin{abstract}
The work process is understood to do, ie put it into practice. As the work process in health an enumerated activities, along with the professional team or alone conducts its social, this work can be physical, intellectual, or even a combination of the two to change or transform something or something. Therefore, this study aimed to Make literature review on the work process in health in primary care. This study it is literature that followed the following steps: choice of topic, research papers published in national scientific journals and electronic libraries available on the Scientific Electronic Library Online (SciELO), and (Dedalus), preparation of the plan work, preparation of the text, for both took into account the Code of Ethics for Professional Nursing. Health work is concurrently related to teamwork, because it requires a collective effort of the people and to develop together a working professional, all staff need to relate and from there develop projects for a good service. Therefore, the organization of the work process in health in primary care enough to promote bonding and accountability with the user services, favoring seeking care when needed.
\end{abstract}

Keywords: Primary Health Care; Process Assessment; Family Health; Care Model. Health 


\section{CONSIDERAÇÕES INICIAIS}

O processo de trabalho em saúde é um tema que está sendo muito discutido ultimamente, principalmente o processo de trabalho na atenção primária, isso tudo por conta dos crescentes números de unidades de saúde da família, que vem sendo construídos ao longo dos dias.

$\mathrm{Na}$ atenção primária, é preciso destacar o processo de trabalho que é essencial para melhorar qualidade do atendimento do profissional de saúde que trabalha nesse nível de atenção, visto que o profissional de saúde nessa área explora mais as tecnologias leves e as tecnologias levesduras.

No atual momento de desenvolvimento da atenção primária o Ministério da saúde aprova a Política Nacional de Atenção Básica que elabora a revisão de diretrizes e normas para organização da atenção primária para a Estratégia Saúde da Família. Essa estratégia foi elaborada para reorganizar e fortalecer a atenção primária como o a porta de entrada a atenção primária à saúde do Sistema Único de Saúde (SUS), através do acesso, a qualificação e a reorientação das práticas de saúde (SOUSA; HAMANN, 2009).
Portanto, a Estratégia Saúde da Família reorienta o Modelo de Atenção e contribui para a consolidação dos princípios do SUS a partir da mudança de visão da saúde atuando na promoção, proteção e recuperação da saúde do individuo, família e comunidade. Além de reordenar a oferta de serviços de saúde alicerçada nos princípios de integralidade, equidade e universalidade, sendo fundamental que se conheça o processo de trabalho em saúde desse nível de atenção para que se possa aprimorar esse novo Modelo de Atenção (NASCIMENTO; NASCIMENTO, 2005).

Deste modo, partindo da necessidade de publicações sobre processo de trabalho âmbito da atenção primária, observou-se o interesse do pesquisador pela temática através do Curso de Pós- Graduação Lato Sensu intitulada "Saúde da Família". Visualizando que quanto melhor o processo de trabalho na atenção primaria melhor à qualidade da assistência prestada aos usuários dessa atenção. Diante disto, o presente estudo teve como objetivo Realizar uma revisão da literatura sobre processo do trabalho em saúde na atenção primária.

\section{CAMINHO METÓDOLOGICO}


Para atingir o objetivo proposto foi realizada uma revisão da literatura. A vantagem deste tipo de estudo reside no fato de permitir investigar uma ampla gama de fenômenos por meio de pesquisa em materiais já elaborados, possibilitando o aprimoramento de idéias e conceitos.

\section{Segundo Martins e Theophilo} (2009), a revisão da literatura é um tipo de estudo que procura explicar e discutir o tema, com base em referências já publicadas anteriormente, buscando sempre conhecer, analisar e explicar as seguintes contribuições para um determinado tema.

Para tanto, escolheu-se inicialmente a temática a ser trabalhada levando-se em consideração a afinidade pela mesma. Pois, sabe-se que a escolha do tema é de importância na elaboração do projeto e requer bastante habilidade dos pesquisadores. $\mathrm{Na}$ escolha do tema os pesquisadores levaram em consideraram o interesse próprio e aptidão na área pesquisada, além de escasso material publicado.

Posteriormente, pesquisou-se de modo não exaustivo artigos publicados em periódicos científicos nacionais e bibliotecas eletrônicas disponíveis na Scientific Electronic Library Online (Scielo), e (Dedalus), que disponibilizaram periódicos nacionais com artigos pertinentes ao tema, sendo eles: Ciência \& Saúde Coletiva, Cadernos de saúde Pública, Rev Esc Enferm USP. Foram utilizados os artigos publicados entre os anos de 2005 à 2012 que apresentaram pelo menos um dos seguintes descritores: Saúde da Família, Atenção Primária à Saúde Programa Saúde da Família, Modelo de Atenção, que estivesse relacionado com a temática trabalhada (Processo de Trabalho na Atenção Primária), pesquisados entre os meses de setembro a novembro de 2012.

Em seguida, elaborou-se um plano de trabalho, fez-se um esboço da estrutura do estudo determinando o que seria abordado com introdução, desenvolvimento e conclusão. A partir daí, elaborou-se o texto com as informações pertinentes ao tema, desenvolvendo o encadeamento dado ao tratamento do tema.

É importante destacar que os pesquisadores levaram em consideração as observâncias éticas contempladas no Código de Ética dos Profissionais de Enfermagem Resolução 311/2007 do Conselho Federal de Enfermagem, no que se refere à elaboração de trabalhos científicos, como mostra os artigos a seguir:

1. "Art. $88-$ Ter reconhecida sua autoria ou participação em produção técnico-científica." 


\section{RESPONSABILIDADES E DEVERES}

2. "[..]. Art. 91 - Respeitar os princípios da honestidade e fidedignidade, bem como os direitos autorais no processo de pesquisa, especialmente na divulgação dos seus resultados.

3. Art. 92 - Disponibilizar os resultados de pesquisa à comunidade científica e sociedade em geral.

4. Art. 93 - Promover a defesa e o respeito aos princípios éticos e legais da profissão no ensino, na pesquisa e produções técnico-científicas."

(COFEN, 2009)

\section{FUNDAMENTAÇÃO TEÓRICA}

O trabalho desde o inicio, é utilizado para realizar necessidade do ser humano através da modificação de materiais da natureza. Deste modo o ser humano em si sentiu-se realizado e tornou-se um ser social a partir do trabalho.

Nesse contexto, o processo de trabalho, é compreendido como o fazer, isto é, o pôr em prática. Sendo o processo de trabalho em saúde um enumerado de atividades, que o profissional juntamente com a equipe ou sozinho realiza atividades de cunho social, esse trabalho pode ser físico, intelectual, ou ainda a junção das duas para mudar ou transformar algo ou alguma coisa. Quando isso é direcionado para atenção primária o processo de trabalho busca realizar proteção, promoção, recuperação e reabilitação do individuo, família e comunidade para de saúde utiliza-se das tecnologias leve, leve- dura e dura (SOUSA; HAMANN, 2009).

Pode-se ainda entender o processo de trabalho em saúde como um modo especifico de trabalhar, onde o produto é consumido no ato da produção, visto como trabalho vivo em ato. Nessa construção do trabalho vivo em ato existe um jogo, onde a negociação determina a autonomia maior ou menor no agir dos profissionais e suas equipes (TAKEMOTO; SILVA, 2007).

O processo de trabalho na atenção primária está voltado para a comunidade com grupos sociais específicos e para isso necessita em seus serviços um alto grau de conhecimento. Desta forma, as tecnologias utilizadas nesse modelo de atenção usam recursos de baixa densidade e de alta complexidade, visto que os recursos utilizados são de baixo custo e que acrescentam instrumentos da tecnologia das ciências sociais e humanas (SOUSA; HAMANN, 2009). 
É imperioso destacar que o processo de trabalho na atenção primária é essencial para melhorar qualidade do atendimento, visto que o profissional nessa área explora mais a tecnologia leve e a tecnologia leve- dura.

Estando a ESF inserido na atenção primária, seu processo de saúde é caracterizado por trabalhar com equipes multiprofissionais com no mínimo um médico, um enfermeiro, um auxiliar de enfermagem ou técnico de enfermagem e seis agentes comunitários de saúde que são responsáveis por atenderem em média de 3.000 habitantes. Para isso, trabalham quarenta horas semanais, perpassando pela intersetorialidade e gestão democrática/ participativa e, sobretudo, pela singularidade dos agentes comunitários de saúde que já são da comunidade e fazem integram a equipe de saúde. Desta forma, tendo intenção de melhorar a forma de pensar e de fazer no cotidiano das famílias nos aspectos de promoção, prevenção, recuperação e reabilitação de doenças e agravos (GIL, 2006).

O trabalho em saúde na ESF em sua maioria que é realizado por diversos profissionais e diversos outros grupos de trabalhadores que realizam diversas tarefas para a manutenção da estrutura institucional da saúde, sendo esse um trabalho assalariado. (SOUSA; HAMANN, 2009)

Portanto, o trabalho em saúde está concomitantemente relacionado ao trabalho em equipe, pois o trabalho em equipe exige das pessoas um trabalho coletivo para desenvolverem junto um trabalho profissional, em que todos da equipe possam se relacionar e a partir daí desenvolverem planejamento de ações.

No cerne da ESF encontram-se problemas existentes que dificulta cada vez mais a assistência nos serviços de saúde pela forma inadequada às demandas por saúde, o que pode ser visto através de poucas ações de promoção e proteção aos usuários, aumentando a insatisfação e insegurança dos usuários com o tipo de assistência prestada (NASCIMENTO; NASCIMENTO, 2005)

Esses problemas estão atrelados segundo Souza (2008) ao acesso desorganizado e fundamentado na demanda espontânea do serviço. Desta forma, os profissionais não conseguem correlacionar as suas especificidades a sua capacidade de resolução, em que o trabalho é operacionalizado sem organização da ação do profissional e acabam sem responder as expectativas e necessidades da população. 
Para Nascimento e Nascimento (2005) é necessário que se conheça as famílias cadastradas nas áreas adscritas, identificando as áreas de riscos e grupos vulneráveis para, a partir disso, juntamente com a população elaborar um plano de ações baseado nas reais necessidades da comunidade, reorganizando as práticas de saúde priorizando as problemáticas elencadas em conjunto utilizando o instrumento de gerência para desenvolver a programação local de saúde.

Porém para desenvolver tais ações planejadas, as equipes de saúde precisam utilizar o acolhimento como estratégia, e estes se dão através de um bom atendimento e da criação do vínculo. Atendimento este que às vezes é bastante difícil nas unidades básicas de saúde, pois a demanda é enorme para um número muito pequeno de profissionais e com isso eles acabam se tornando atarefados (SOUSA; HAMANN, 2009).

\section{A intenção do Ministério da} Saúde quando implantou a ESF era diminuir a demanda de usuários nos prontos atendimentos, mas isso foi só mera ilusão, pois os locais de emergência ainda continuam cheios, uma vez que os usuários preferem esse local de atendimento, pois nele o atendimento é mais rápido do que as unidades que as vezes nem profissional tem. (BARROS; SÁ, 2010)
Para alcançar o maior sucesso o processo de trabalho tem uma visão ampla de melhoria dos serviços de saúde e essa visão está voltada na assistência básica. Porém, as unidades de saúde ainda não estão com base nesse processo, pois elas estão se tornado um modelo de trabalho em equipe com comunicação adequada e troca de experiências, não sendo apenas um trabalho coletivo com ênfase na humanização da assistência e na ética da responsabilidade.

À medida que vencesse $\mathrm{o}$ processo relacional entre o trabalhador de saúde e o usuário haverá uma dimensão individual do trabalho em saúde. É nessa dimensão no processo de trabalho em saúde que envolve o acolhimento adquire uma expressão significativa. Nesse contexto, operam-se tecnologias leves e com isso o trabalho vivo em ato entra em favor do bom atendimento, onde se destacam relações de escuta, responsabilizações, criação de vínculos que buscam agir com base nas necessidades, buscando a produção de algo. (FRANCO et al., 2005)

Diante desse contexto, o trabalho vivo em ato na unidade básica de saúde da família, vem sendo muito realizado e com isso melhorando o atendimento para com os usuários que necessitam de certa ajuda, com enfoque na prevenção, promoção, recuperação 
e reabilitação da saúde permitindo que a família sofra menos agravo à saúde.

O acolhimento como diretriz operacional tem os seguintes princípios:

- Atendimento universal com garantia na acessibilidade

- Reorganizar o processo de trabalho retirando o foco central do médico e divergindo o foco para a equipe multiprofissional

- Melhorar a relação usuário profissional de saúde dando ênfase na humanização, solidariedade e cidadania (FRANCO et al., 2005).

No entanto, devido à grande pressão exercida pelo déficit de profissionais e materiais, acabam não respondendo a expectativa do usuário da atenção primária, gerando desta maneira longas filas de esperas para alguns atendimentos, outra dificuldade é a menor resolutividade de problemas na rede primária, em que o profissional muitas vezes não esgota as possibilidades de resolver o problema antes de encaminhar esse usuário para um serviço especializado gerando gastos desnecessários isso mostra o modo de operar o trabalho (SOUZA et al.,2008)

Para o autor supracitado o que prevalece na atenção primária é processo de trabalho em que os profissionais esquecem-se da subjetividade dos sujeitos que apresentam histórias de vida e que essas que isso também é um determinante no processo de adoecimento.

Compreendendo que a Estratégia de Saúde da Família tem como base, o acolhimento, o vínculo e a responsabilização, destacou-se que existe uma dificuldade de vínculo com as pessoas de nível social mais alto. O que faz as pessoas apontarem para o serviço segundo Barros e Sá (2010) é a necessidade da utilização do mesmo, deste modo por essas pessoas não sentirem que necessitam da unidade não permite a criação de vínculos.

Destarte que para um processo de trabalho eficiente na ESF é imperioso que coexista o vinculo e acolhimento juntamente com a responsabilização de esgota-se as possibilidades de resolver os problemas. Acrescido a isso é preciso dirimir o déficit de profissionais e materiais que gera longas filas de esperas para alguns atendimentos, respondendo assim a expectativa do usuário da atenção primária.

\section{CONSIDERAÇÕES FINAIS}

A organização do processo em saúde deve promover o vínculo e 
responsabilização com o usuário dos serviços, favorecendo a busca de atendimento quando necessário. Deste modo, percebe a necessidade urgente da construção de um novo modelo assistencial de saúde na Estratégia Saúde da Família, pautado pelas tecnologias leves e leve-duras, sobretudo. No entanto, essa estratégia ainda encontra muito desafios.

É indispensável que o processo de trabalho seja baseado em condições sóciopolíticas, materiais e recursos humanos existentes no ambiente de trabalho, para que assim possibilite melhorias na qualidade do cuidado para quem assiste e para quem é assistido na ESF.

Ainda levando em consideração a construção desse modelo é fundamental a humanização do atendimento desde a porta de entrada à porta de saída dos serviços de saúde, permitindo uma maior criação de vínculo e resolubilidade das necessidades.

Assim, a organização do processo de trabalho em saúde na atenção primária é de uma importância imensurável, tendo em vista que é um dos locais mais freqüentados pelos usuários dos serviços de saúde, devido ser acesso aos demais setores da saúde, dos mais básicos aos mais complexos. Mas, para alcançar tal proposta é essencial que a discussão e o trabalho intenso e ininterrupto acerca do tema atualmente. Para que em um futuro próximo possamos visualizar alguns frutos da proposta da utilização das tecnologias leves e leve-duras, da capacitação profissional e do envolvimento dos usuários como instrumento de fortalecimento do processo de trabalho em saúde.

\section{REFERÊNCIAS}

BARROS, D. M.; SÁ, M. C. O processo de trabalho em saúde e a produção do cuidado em uma unidade de saúde da família: límites ao acolhimento e reflexos no serviço de emergência. Ciência \& Saúde Coletiva: v.15, n.5, pp.2473-2482, 2010.

FRANCO, T. B.; MAGALHÃES JÚNIOR, H. M. Integralidade na assistência à saúde: A organização de linhas de cuidado. IN:

MERHY, E. E. et al. O trabalho em saúde: olhando e experienciando o SU S no cotidiano. 2 ed. São Paulo: HUCITEC, 2005

GIL, C. R. R.. Atenção primária, atenção básica e saúde da família: sinergias e singularidades do contexto brasileiro.Cad. Saúde Pública: Rio de Janeiro, v.2, n.6, pp.1171-1181, 2006.

MARTINS, G.A.; THEÓPHILO, C. R. Metodologia da investigação científica para ciências sociais aplicadas. São Paulo: Atlas, 2009.

NASCIMENTO, M. S.; NASCIMENTO, M. A. A. Prática da enfermeira no Programa de Saúde da Família: a interface da vigilância da saúde versus as ações programáticas em saúde. Ciência \& Saúde Coletiva: v. 10, n.2, pp. 333-345, 2005. 
SOUSA, M. F.; HAMANN, E. M. Programa

Saúde da Família no Brasil: uma agenda

incompleta?. Ciência \& Saúde Coletiva:

v.14, n.1, pp.1325-1335, 2009.

SOUZA, E. C. F. Acesso e acolhimento na atenção básica: uma análise da percepção dos usuários e profissionais de saúde. Cad. Saúde Pública: v. 24, n. 1, pp. S100-S110, 2008.

TAKEMOTO, M. L S.; SILVA, E. M. Acolhimento e transformações no processo de trabalho em enfermagem em unidades básicas de saúde de Campinas, São Paulo, Brasil.

Cad. Saúde Pública: Rio de Janeiro, v. 23, n.2, pp. 331-340,2007. 